\title{
Unimodular integer circulants associated with trinomials
}

\author{
Gerald Williams
}

May 8, 2009

\begin{abstract}
The $n \times n$ circulant matrix associated with the polynomial $f(t)=\sum_{i=0}^{d} a_{i} t^{i}$ (with $d<n$ ) is the one with first row $\left(a_{0} \ldots a_{d} 0 \ldots 0\right)$. The problem as to when such circulants are unimodular arises in the theory of cyclically presented groups and leads to the following question, previously studied by Odoni and Cremona: when is $\operatorname{Res}\left(f(t), t^{n}-1\right)= \pm 1$ ? We give a complete answer to this question for trinomials $f(t)=t^{m} \pm t^{k} \pm 1$. Our main result was conjectured by the author in an earlier paper and (with two exceptions) implies the classification of the finite Cavicchioli-Hegenbarth-Repovš generalized Fibonacci groups, thus giving an almost complete answer to a question of Bardakov and Vesnin.
\end{abstract}

\section{Introduction}

The $n \times n$ circulant matrix $M_{n}(f)$ associated with the polynomial $f(t)=\sum_{i=0}^{d} a_{i} t^{i}$ where $d<n$ and $a_{i} \in \mathbb{Z}$ is the one whose first row is $\left(a_{0} \ldots a_{d} 0 \ldots 0\right)$. Well known properties of circulants and resultants give that $\operatorname{det}\left(M_{n}\right)=\operatorname{Res}\left(f, t^{n}-1\right)$. The question as to when $M_{n}$ is unimodular arises in the theory of cyclically presented groups and has been considered by Odoni [7] and Cremona [3].

For $n \geq 1$ define

$$
R_{n}(f)=\prod_{\theta^{n}=1} f(\theta) .
$$

Our approach, as in [3],[7], is to work with $R_{n}(f)$ rather than with $M_{n}(f)$. It was shown in [3],[7] that, for $n>d, \operatorname{det}\left(M_{n}\right)=R_{n}(f)$ so it is enough to consider when $R_{n}(f)= \pm 1$. We note that $R_{n}(f)$ is defined for all $n \geq 1$ whereas $M_{n}(f)$ is only defined for $n>d$.

Briefly, the connection with cyclically presented groups is as follows. Fix a word $w\left(x_{0}, \ldots, x_{n-1}\right)$ in generators $x_{0}, \ldots, x_{n-1}$ and let $\Gamma_{n}(w)$ be the group defined by the presentation with these $n$ generators and the $n$ relators

$$
w\left(x_{0}, x_{1}, \ldots, x_{n-2}, x_{n-1}\right), w\left(x_{1}, x_{2}, \ldots, x_{n-1}, x_{0}\right), \ldots, w\left(x_{n-1}, x_{0}, \ldots, x_{n-3}, x_{n-2}\right) .
$$

If $a_{i}$ is the exponent sum of $x_{i}$ in $w\left(x_{0}, \ldots, x_{n-1}\right)$ then $\Gamma_{n}(w)$ has infinite abelianization if and only if $R_{n}(f)=0$ and is perfect if and only if $R_{n}(f)= \pm 1$ [5],[7]. Indeed $\Gamma_{n}(w)^{\text {ab }}$ has order $\left|R_{n}(f)\right|$ ([5, page 77]). 
In this paper we consider trinomials $f(t)=t^{m} \pm t^{k} \pm 1$. When both signs are ' + ' it is easy to deduce that $R_{n}(f) \neq \pm 1$. In the other three cases we can reduce to a polynomial of the form $t^{m}-t^{k}+1$; moreover we may assume $(n, m, k)=1$ (see Section 3). We note that Lemma 5 of [8] and Lemma 2.3 of [4] determine when $R_{n}\left(t^{m} \pm t^{k} \pm 1\right)=0$.

The Cavicchioli-Hegenbarth-Repovš generalized Fibonacci groups $G_{n}(m, k)$ are the cyclically presented groups with generators $x_{1}, \ldots, x_{n}$ and relators $x_{i} x_{i+m} x_{i+k}^{-1}$ and these are our primary motivation for considering trinomials $f(t)=t^{m}-t^{k}+1$. Our main result is

Main Theorem Let $n \geq 1$ and $f(t)=t^{m}-t^{k}+1$ where $m, k \in \mathbb{Z}$ and $(n, m, k)=$ 1. Then $R_{n}(f)= \pm 1$ if and only if $((n, 6)=1$ and $m=2 k \bmod n)$ or $k=0 \bmod n$ or $k=m \bmod n$.

This was conjectured (in group theoretic terms) in [8] and is a natural generalization of a theorem of Odoni [7] which deals with the case $k=1$. With the exception of two groups, the Main Theorem implies the classification of the finite groups $G_{n}(m, k)$ (see [8]), thus giving an almost complete answer to a problem posed by Bardakov and Vesnin ([1, Question 1]).

\section{Preliminaries}

A number of equivalent characterizations of $R_{n}(f)= \pm 1$ were given in [3],[7]. We only need some of them:

Lemma $2.1([3,7])$ For $f \in \mathbb{Z}[t]$ and $n \geq 1$ the following are equivalent:

(a) $R_{n}(f)= \pm 1$;

(b) $f\left(\zeta_{d}\right)$ is a unit in the ring $\mathbb{Z}\left[\zeta_{d}\right]$ for all $d \mid n$ where $\zeta_{d}$ denotes a primitive dth root of unity;

(c) $\operatorname{Res}\left(f, t^{n}-1\right)= \pm 1$.

We record some properties of $R_{n}$; those in Proposition 2.2 follow directly from its definition.

Proposition 2.2 Let $f, g \in \mathbb{Z}[t]$ and let $n \geq 1$. Then the following hold:

(a) $R_{n}(f g)=R_{n}(f) R_{n}(g)$;

(b) $R_{n}(t)=(-1)^{n+1}$;

(c) If $m \mid n$ then $R_{m}(f) \mid R_{n}(f)$. 
Proposition $2.3([7])$ Let $f(t)=c \prod_{j=1}^{k}\left(t-\beta_{j}\right)$. Then

$$
R_{n}(f)=\left((-1)^{k} c\right)^{n} \prod_{j=1}^{k}\left(\beta_{j}^{n}-1\right) .
$$

In [3] the expression $c^{n} \prod_{j=1}^{k}\left(\beta_{j}^{n}-1\right)$ was denoted $B(f, n)$ and so $R_{n}(f)= \pm B(f, n)$.

Proposition 2.4 Let $f, F \in \mathbb{Z}[t]$ be polynomials such that $f(t)=F\left(t^{\alpha}\right)$ for some $\alpha \in$ $\mathbb{N}$. Then

$$
R_{n}(f)=\left(R_{n /(n, \alpha)}(F)\right)^{(n, \alpha)} .
$$

In particular $R_{n}\left(t^{m} \pm t^{k} \pm 1\right)=\left(R_{N}\left(t^{M} \pm t^{K} \pm 1\right)\right)^{(n, m, k)}$ where $N=n /(n, m, k)$, $M=m /(m, k), K=k /(m, k)$.

\section{Proof}

Let $d=(n, \alpha)$. Then we have

$$
R_{n}(f)=\prod_{\theta^{n}=1} F\left(\theta^{\alpha}\right)=\prod_{q=0}^{n-1} F\left(e^{2 \pi i q \alpha / n}\right)=\prod_{q=0}^{n-1} F\left(e^{2 \pi i q(\alpha / d) /(n / d)}\right)
$$

which is equal to

$$
\left(\prod_{q=0}^{n / d-1} F\left(e^{2 \pi i q(\alpha / d) /(n / d)}\right)\right)^{d}
$$

so $R_{n}(f)=\left(R_{n / d}(F)\right)^{d}$. Now since $(\alpha / d, n / d)=1$, for each $q=0, \ldots,(n / d-1)$ there exists a unique $Q=0, \ldots,(n / d-1)$ such that $q(\alpha / d)=Q \bmod n / d$. Hence

$$
\prod_{q=0}^{n / d-1} F\left(e^{2 \pi i q(\alpha / d) /(n / d)}\right)=\prod_{Q=0}^{n / d-1} F\left(e^{2 \pi i Q /(n / d)}\right)=\prod_{\phi^{n / d}=1} F(\phi)=R_{n / d}(F)
$$

so $R_{n}(f)=\left(R_{n / d}(F)\right)^{d}$.

To prove the last claim let $f(t)=t^{m} \pm t^{k} \pm 1$ and $F(t)=t^{M} \pm t^{K} \pm 1$.

Since $(N, M, K)=1$, in considering when $R_{n}\left(t^{m} \pm t^{k} \pm 1\right)= \pm 1$ Proposition 2.4 allows us to assume that $(n, m, k)=1$.

\section{Properties of $R_{n}\left(t^{m} \pm t^{k} \pm 1\right)$}

We have that $R_{1}\left(t^{m}+t^{k}+1\right)=3$ so by Proposition 2.2 (c) $R_{n}\left(t^{m}+t^{k}+1\right) \neq \pm 1$ for all $n$. Thus we may assume that at least one of the signs is a ' - '.

Proposition $3.1 \quad$ (a) $\left|R_{n}\left(t^{m}-t^{k}-1\right)\right|=\left|R_{n}\left(t^{k}-t^{m}+1\right)\right|$; 
(b) $\left|R_{n}\left(t^{m}+t^{k}-1\right)\right|=\left|R_{n}\left(t^{k-m}-t^{k}+1\right)\right|$;

(c) $\left|R_{n}\left(t^{m}-t^{k}+1\right)\right|=\left|R_{n}\left(t^{m}-t^{m-k}+1\right)\right|$.

\section{Proof}

(a) $t^{m}-t^{k}-1=-\left(t^{k}-t^{m}+1\right)$ so $\left|R_{n}\left(t^{m}-t^{k}-1\right)\right|=\left|R_{n}\left(t^{k}-t^{m}+1\right)\right|$.

(b) $t^{m}+t^{k}-1=t^{k}\left(t^{m-k}-t^{-k}+1\right)$ so

$$
\begin{aligned}
R_{n}\left(t^{m}+t^{k}-1\right) & =R_{n}\left(t^{k}\right) R_{n}\left(t^{m-k}-t^{-k}+1\right) \\
& =\left(R_{n}(t)\right)^{k} R_{n}\left(t^{k-m}-t^{k}+1\right) \\
& = \pm R_{n}\left(t^{k-m}-t^{k}+1\right) .
\end{aligned}
$$

(c) $t^{m}-t^{k}+1=t^{m}\left(t^{-m}-t^{k-m}+1\right)$ so

$$
\begin{aligned}
R_{n}\left(t^{m}-t^{k}+1\right) & =R_{n}\left(t^{m}\right) R_{n}\left(t^{-m}-t^{k-m}+1\right) \\
& =\left(R_{n}(t)\right)^{m} R_{n}\left(t^{m}-t^{m-k}+1\right) \\
& = \pm R_{n}\left(t^{m}-t^{m-k}+1\right) .
\end{aligned}
$$

Other similar identities can be established. For example [2, Theorem 2] implies that if $n, m, k, m^{\prime}, k^{\prime}$ are integers such that $(n, m, k)=1,\left(n, m^{\prime}, k^{\prime}\right)=1,\left(n, k^{\prime}\right)=1$ and $m^{\prime}(m-k)=m k^{\prime} \bmod n$ then $R_{n}\left(t^{m}-t^{k}+1\right)= \pm R_{n}\left(t^{m^{\prime}}-t^{k^{\prime}}+1\right)$.

Parts (a) and (b) of Proposition 3.1 show that $R_{n}\left(t^{m}-t^{k}-1\right)= \pm R_{n}\left(t^{m^{\prime}}-\right.$ $t^{k^{\prime}}+1$ ) (for some $\left.m^{\prime}, k^{\prime}\right)$ and $R_{n}\left(t^{m}+t^{k}-1\right)= \pm R_{n}\left(t^{m^{\prime}}-t^{k^{\prime}}+1\right.$ ) (for some $m^{\prime}, k^{\prime}$ ), so we only need consider $R_{n}(f)$ for $f(t)=t^{m}-t^{k}+1$. Moreover, by Proposition 2.4 we may assume that $(n, m, k)=1$. Proposition 3.1(c) shows that the roles of $k$ and $(m-k)$ can be interchanged.

The next result was prompted by [1, Lemma 1.3].

Proposition 3.2 (a) If $(k, n)=1$ then $R_{n}\left(t^{m}-t^{k}+1\right)=R_{n}\left(t^{m \ell}-t+1\right)$ where $\ell=k^{-1} \bmod n$;

(b) if $(m-k, n)=1$ then $R_{n}\left(t^{m}-t^{k}+1\right)=R_{n}\left(t^{m \ell}-t+1\right)$ where $\ell=(m-$ $k)^{-1} \bmod n$;

(c) if $(m, n)=1$ then $R_{n}\left(t^{m}-t^{k}+1\right)=R_{n}\left(t-t^{k \ell}+1\right)$ where $\ell=m^{-1} \bmod n$.

\section{Proof}

(a) Let $\phi=\theta^{k}$, then $\theta=\phi^{\ell}$ so

$$
R_{n}\left(t^{m}-t^{k}+1\right)=\prod_{\theta^{n}=1} \theta^{m}-\theta^{k}+1=\prod_{\phi^{n}=1}\left(\phi^{\ell}\right)^{m}-\phi+1=R_{n}\left(t^{m \ell}-t+1\right) .
$$


(b) This follows from (a) by interchanging the roles of $k$ and $(m-k)$.

(c) Similar to (a).

Parts (a),(b) of Proposition 3.2 show that it is sometimes enough to consider the polynomials considered by Odoni [7] (that is, polynomials of the form $t^{m}-t+1$ ).

When $k=0 \bmod n$ or $k=m \bmod n$ it is clear that $R_{n}\left(t^{m}-t^{k}+1\right)= \pm 1$. We can obtain the value of $R_{n}$ in some other cases; for example by Proposition 2.3 and Proposition 2.4 we have that $R_{n}\left(t^{0}-t^{k}+1\right)=\left(2^{n /(n, k)}-1\right)^{(n, k)}$. By [8, Lemma 3] we also have

Lemma 3.3 Suppose that $n$ is even, $(m, k)=1$ and either $k=n / 2 \bmod n$ or $(m-k)=n / 2 \bmod n$. Then $\left|R_{n}\left(t^{m}-t^{k}+1\right)\right|=2^{n / 2}-(-1)^{m+n / 2}$.

\section{Proof of Main Theorem}

Odoni proved the Main Theorem in the case $k=1$ : we summarize this result ([7, Theorem 2(ii),(iii)]) as

Theorem 4.1 ([7]) Let $n \geq 1$ and $f(t)=t^{m}-t+1$ where $m \in \mathbb{Z}$. Then $R_{n}(f)=$ \pm 1 if and only if $((n, 6)=1$ and $m=2 \bmod n)$ or $m=1 \bmod n$.

Corollary 4.2 Let $n \geq 1$ and $f(t)=t^{m}-t^{k}+1$ where $m, k \in \mathbb{Z},(n, m, k)=1$ and suppose that either $(k, n)=1$ or $(m-k, n)=1$. Then $R_{n}(f)= \pm 1$ if and only if $((n, 6)=1$ and $m=2 k \bmod n)$ or $k=0 \bmod n$ or $k=m \bmod n$.

\section{Proof}

By interchanging the roles of $k$ and $(m-k)$ we may assume that $(k, n)=1$. By Proposition 3.2(a) $R_{n}(f)=R_{n}\left(t^{m \ell}-t+1\right)$, where $\ell=k^{-1} \bmod n$. Now $m \ell=1,2 \bmod n$ if and only if $m=k, 2 k \bmod n$, so the result follows from Theorem 4.1 .

The following corollary generalizes [7, Lemma 3.2] to our setting.

Corollary 4.3 Let $n=p^{u}$ where $p=2$ or $3, u \geq 1$, and $f(t)=t^{m}-t^{k}+1$ where $m, k \in \mathbb{Z},(n, m, k)=1$. Then $R_{n}(f)= \pm 1$ if and only if $k=0 \bmod n$ or $k=m \bmod n$.

\section{Proof}

The hypotheses imply that either $(k, n)=1$ or $(m-k, n)=1$ and so the result follows from Corollary 4.2 .

The 'if' direction of the Main Theorem is straightforward to prove (see $[8$, Lemma 5]) so from now on we focus on the 'only if' direction. 
Lemma 4.4 Let $n=2^{r} 3^{s} \geq 1$ and $f(t)=t^{m}-t^{k}+1$ where $m, k \in \mathbb{Z},(n, m, k)=1$. If $R_{n}(f)= \pm 1$ then $k=0 \bmod n$ or $k=m \bmod n$.

\section{Proof}

By Corollary 4.3 we may assume $r \geq 1, s \geq 1$. Now $R_{n}(f)= \pm 1$ implies $R_{2^{r}}(f)=$ \pm 1 and so by Corollary 4.3 we have $k=0 \bmod 2^{r}$ or $(m-k)=0 \bmod 2^{r}$. By interchanging the roles of $k$ and $(m-k)$ we may assume that the first of these holds. We also have $R_{3^{s}}(f)= \pm 1$ so $k=0 \bmod 3^{s}$ or $k=m \bmod 3^{s}$. In the first case we have $k=0 \bmod n$, so assume the second.

Let $d=2 \cdot 3^{s}$. Now $k \neq m \bmod d$, for otherwise $2 \mid(n, m, k)=1$; thus $k=m+d / 2 \bmod d$. It follows that $(m \bmod d, k \bmod d)=1$ so Lemma 3.3 implies that $R_{d}(f) \neq \pm 1$ and hence $R_{n}(f) \neq \pm 1$.

Our next lemma generalizes [7, Lemma 3.3] to our setting. We use ideas from the proof of that result.

Lemma 4.5 Let $n=p q$ where $q=2$ or 3 and $p \geq 5$ is prime, $f(t)=t^{m}-t^{k}+1$ where $m, k \in \mathbb{Z},(n, m, k)=1$. If $R_{n}(f)= \pm 1$ then $k=0 \bmod n$ or $k=m \bmod n$.

\section{Proof}

By Corollary 4.2 we may assume $(k, n)>1,(m-k, n)>1$ so $($ since $(n, m, k)=1)$ either $(q \mid k$ and $p \mid(m-k))$ or $(p \mid k$ and $q \mid(m-k))$. By interchanging the roles of $k$ and $(m-k)$ we may assume that the first case occurs, i.e. $k=0 \bmod q$ and $(m-k)=0 \bmod p$. Moreover we may assume $k \neq 0 \bmod p,(m-k) \neq 0 \bmod q$ for otherwise $k=0 \bmod n$ or $(m-k)=0 \bmod n$.

If either $p$ or $q$ divides $m$ then we get a contradiction to $(n, m, k)=1$ so $(m, n)=$ 1. Now by Proposition $3.2 R_{n}(f)=R_{n}(g)$ where $g(t)=t^{m^{\prime}}-t^{k^{\prime}}+1$ where $m^{\prime}=1$, $k^{\prime}=k m^{-1}$. The conditions on $m, k$ imply $k^{\prime}=0 \bmod q,\left(m^{\prime}-k^{\prime}\right)=0 \bmod p$, $\left(m^{\prime}-k^{\prime}\right) \neq 0 \bmod q$. When $q=2$ we have that $\left(m^{\prime}-k^{\prime}\right)=n / 2 \bmod n$ and since $\left(m^{\prime}, k^{\prime}\right)=1$ Lemma 3.3 implies $R_{n}(g) \neq \pm 1$.

Suppose then that $q=3$ and $R_{n}(g)= \pm 1$. We have that $k^{\prime}=1 \bmod p$, $k^{\prime}=0 \bmod 3$. Now $R_{3 p}(g)= \pm 1$ so, writing $\zeta_{d}$ for a primitive $d$ th root of unity, Lemma 2.1 implies that $g(\theta)$ is a unit in $\mathbb{Z}\left[\zeta_{3 p}\right]$ whenever $\theta$ is a primitive $(3 p)$ th root of unity. In particular $g\left(\zeta_{p} \zeta_{3}\right)=\zeta_{p}\left(\zeta_{3}-1\right)+1$ and $g\left(\zeta_{p} \zeta_{3}^{2}\right)=\zeta_{p}\left(\zeta_{3}^{2}-1\right)+1$ are units in $\mathbb{Z}\left[\zeta_{3 p}\right]$ and hence so is their product $3 \zeta_{p}^{2}-3 \zeta_{p}+1$, which must therefore also be a unit in $\mathbb{Z}\left[\zeta_{p}\right]$. Let $h(x)=3 x^{2}-3 x+1$. Then $h(1)=1$ and $h\left(\zeta_{p}\right)$ are units in $\mathbb{Z}\left[\zeta_{p}\right]$ so by Lemma 2.1 we have that $R_{p}(h)= \pm 1$. Now Proposition 2.3 implies that $R_{p}(h)=3^{p}\left(\beta_{1}^{p}-1\right)\left(\beta_{2}^{p}-1\right)$ where $\beta_{1}, \beta_{2}=3^{-1 / 2} e^{ \pm i \pi / 6}$ are the roots of $h$. But

$$
3^{p}\left(\beta_{1}^{p}-1\right)\left(\beta_{2}^{p}-1\right)=3^{p}+1 \pm 3^{(p+1) / 2} \neq \pm 1
$$

and we have a contradiction. 
Our next result (Lemma 4.8) deals with the case $(n, 6)=1$. It generalizes $[7$, Lemma 3.1] to our setting and its proof is essentially a re-run of the proof of that result. We will require the following theorem of Kronecker, a proof of which can be found on page 46 of [6].

Lemma 4.6 Let $\beta=\beta_{1}$ be a non-zero algebraic integer and let $\beta_{1}, \ldots, \beta_{k}$ be the conjugates of $\beta$ over $\mathbb{Q}$. If $\max _{j}\left|\beta_{j}\right| \leq 1$ then $\beta$ is a root of unity.

We will also need the following:

Lemma 4.7 If $\sum_{i=1}^{\ell} w_{i}^{j}=\sum_{i=1}^{\ell} z_{i}^{j}$ for all $j=1, \ldots, \ell$ then the multisets $\left\{w_{1}, \ldots, w_{\ell}\right\}$ and $\left\{z_{1}, \ldots, z_{\ell}\right\}$ are equal.

The proof is a standard application of the Newton-Girard formula and so is omitted.

Lemma 4.8 Let $n \geq 1$ and $f(t)=t^{m}-t^{k}+1$ where $m, k \in \mathbb{Z},(n, m, k)=1$ and suppose $(n, 6)=1$. If $R_{n}(f)= \pm 1$ then $m=2 k \bmod n$ or $k=0 \bmod n$ or $k=m \bmod n$.

\section{Proof}

By Lemma $2.1 \lambda=f(\zeta)$ is a unit in the ring $\mathbb{Z}[\zeta]$ for some primitive $n$th root of unity $\zeta$, and therefore so is $\sigma(\lambda)$ for any $\sigma \in \Gamma=\operatorname{Gal}(\mathbb{Q}(\zeta) / \mathbb{Q})$. Let $\mu=\bar{\lambda} \lambda^{-1}$. Then, since $\Gamma$ is abelian, we have

$$
|\sigma(\mu)|^{2}=\sigma(\mu) \overline{\sigma(\mu)}=\sigma(\mu \bar{\mu})=\sigma(1)=1 .
$$

Lemma 4.6 implies that $\mu$ is a root of unity in $\mathbb{Q}(\zeta)$, and thus $\mu=s \zeta^{j}$ for some $j \in \mathbb{Z}, s= \pm 1$. Since $\mu=\bar{\lambda} \lambda^{-1}=\overline{f(\zeta)} f(\zeta)^{-1}=f\left(\zeta^{-1}\right) f(\zeta)^{-1}$ it follows that

$$
s \zeta^{j}\left(\zeta^{m}-\zeta^{k}+1\right)=\zeta^{-m}-\zeta^{-k}+1 .
$$

Case 1: $s=-1$. Let $w_{1}=\zeta^{-m}, w_{2}=1, w_{3}=\zeta^{m+j}, w_{4}=\zeta^{j}, z_{1}=\zeta^{-k}, z_{2}=$ $\zeta^{j+k}, z_{3}=z_{4}=0$. Then (4.1) is equivalent to

$$
w_{1}+w_{2}+w_{3}+w_{4}=z_{1}+z_{2}+z_{3}+z_{4} .
$$

Since $(n, 6)=1$ we have that $(r, n)=1$ for $r=1,2,3,4$. Thus the maps $\zeta \mapsto \zeta^{r}$ $(r=1,2,3,4)$ are automorphisms of $\mathbb{Q}(\zeta)$. Applying these to $(4.2)$ we get

$$
\sum_{i=1}^{4} w_{i}^{r}=\sum_{i=1}^{4} z_{i}^{r} \quad(r=1,2,3,4) .
$$

By Lemma 4.7 we have that $\left\{w_{1}, w_{2}, w_{3}, w_{4}\right\}=\left\{z_{1}, z_{2}, z_{3}, z_{4}\right\}$, but $z_{3}=0 \notin$ $\left\{w_{1}, w_{2}, w_{3}, w_{4}\right\}$ which gives a contradiction. 
Case 2: $s=+1$. Let $w_{1}=\zeta^{-m}, w_{2}=1, w_{3}=\zeta^{k+j}, z_{1}=\zeta^{-k}, z_{2}=\zeta^{j+m}, z_{3}=\zeta^{j}$. Then (4.1) is equivalent to

$$
w_{1}+w_{2}+w_{3}=z_{1}+z_{2}+z_{3} .
$$

As in Case 1 , the maps $\zeta \mapsto \zeta^{r}(r=1,2,3)$ are automorphisms of $\mathbb{Q}(\zeta)$ and applying them to (4.4) gives $\left\{w_{1}, w_{2}, w_{3}\right\}=\left\{z_{1}, z_{2}, z_{3}\right\}$. If $\left(z_{1}, z_{2}, z_{3}\right)=\left(w_{3}, w_{1}, w_{2}\right)$ then $\zeta^{2 k}=\zeta^{2 m}=1$ so $k=0$ or $n / 2 \bmod n$ and $m=0$ or $n / 2 \bmod n$ and so $k=0$ or $m \bmod n$ or $m=2 k \bmod n$. If $\left(z_{1}, z_{2}, z_{3}\right)=\left(w_{1}, w_{2}, w_{3}\right),\left(w_{1}, w_{3}, w_{2}\right)$, or $\left(w_{2}, w_{3}, w_{1}\right)$ then $\zeta^{m-k}=1$ and hence $k=m \bmod n$. If $\left(z_{1}, z_{2}, z_{3}\right)=\left(w_{3}, w_{2}, w_{1}\right)$ then $2 k=m \bmod n$. If $\left(z_{1}, z_{2}, z_{3}\right)=\left(w_{2}, w_{1}, w_{3}\right)$ then $k=0 \bmod n$.

\section{Proof of Main Theorem}

The 'if' direction was proved in [8, Lemma 5] so suppose that $R_{n}(f)= \pm 1$. By Lemmas 4.4 and 4.8 we may assume $n=a b$ where $a=2^{r} 3^{s}>1,(b, 6)=1, b>1$. Now $R_{a}(f)= \pm 1$ implies (by Lemma 4.4) that $k=0 \bmod a$ or $(m-k)=0 \bmod a$. By interchanging the roles of $k$ and $(m-k)$ we may assume that $k=0 \bmod a$. Also, $R_{b}(f)= \pm 1$ implies (by Lemma 4.8 ) that $k=0 \bmod b$ or $m=2 k \bmod b$ or $k=m \bmod b$. If $k=0 \bmod b$ then $k=0 \bmod n$ so assume otherwise.

Suppose $m=2 k \bmod b$. Then no prime divisor of $n$ divides $(m-k)$ for otherwise it would also divide $(n, m, k)=1$. Therefore $(m-k, n)=1$ and the result follows from Corollary 4.2. Suppose then that $k=m \bmod b$ and let $p \geq 5$ be a prime divisor of $b$ and let $q=2$ if $r \geq 1$ or $q=3$ otherwise. Now $k \neq m \bmod p q$ and $k \neq 0 \bmod p q$ for otherwise $q \mid(n, m, k)=1$ or $p \mid(n, m, k)=1$ (respectively) and so Lemma 4.5 implies $R_{p q}(f) \neq \pm 1$ so $R_{n}(f) \neq \pm 1$.

\section{Acknowledgement}

I would like to thank the referee(s) of this paper and of an earlier version of it for the helpful comments.

\section{References}

[1] V.G. Bardakov and A.Yu. Vesnin. A generalization of Fibonacci groups. Algebra and Logic, 42(2):131-160, 2003.

[2] A. Cavicchioli, E.A. O'Brien, and F. Spaggiari. On some questions about a family of cyclically presented groups. J. Algebra, 320(11):4063-4072, 2008.

[3] J.E. Cremona. Unimodular integer circulants. Math. Comp., 77:1639-1652, 2008. 
[4] M. Edjvet and G. Williams. The cyclically presented groups with relators $x_{i} x_{i+k} x_{i+\ell}$. Preprint.

[5] D.L. Johnson. Topics in the theory of group presentations, volume 42 of London Mathematical Society Lecture Note Series. Cambridge University Press, 1980.

[6] W. Narkiewicz. Elementary and analytic theory of algebraic numbers. SpringerVerlag, second edition, 1990.

[7] R.W.K. Odoni. Some Diophantine problems arising from the theory of cyclically-presented groups. Glasg. Math. J., 41(2):157-165, 1999.

[8] G. Williams. The aspherical Cavicchioli-Hegenbarth-Repovš generalized Fibonnacci groups. J.Group Theory, 12(1):139-149, 2009.

\section{Author's address}

Gerald Williams

Department of Mathematical Sciences

University of Essex

Wivenhoe Park

Colchester

Essex

CO4 3SQ

gwill@essex.ac.uk 